\title{
Les facteurs associés à la satisfaction des citoyens camerounais envers la démocratie
}

\author{
Factors associated with Cameroonian citizens' \\ satisfaction with democracy
}

\author{
Judicaël Alladatin, Universite Mohammed VI Polytechnique, Maroc \\ (judicael.alladatin@um6p.ma) \\ Bekono Nkoudou Hugues, Ministère camerounais des Relations Extérieures \\ (bekono.hugues@diplocam.cm)
}

Résume : Les études sur la satisfaction envers la démocratie ont généralement pour objectif de mesurer/scruter ou juger l'action/les réalisations des décideurs en vue d'améliorer la gouvernance. De notre étude, il ressort que $62 \%$ des citoyens camerounais sont insatisfaits de la mise en œuvre de la démocratie. Cette situation résulte de trois facteurs : la perception de la situation économique du pays, les conditions de vie actuelle des citoyens et leur milieu de résidence (urbain et rural). Mettre un accent sur ces déterminants dans l'élaboration des politiques publiques permettrait sans doute à terme de relever la satisfaction des citoyens et d'assurer le développement du pays.

Mots Clés : satisfaction, facteurs associés, gouvernance, Cameroun

Abstract: Studies on satisfaction with democracy generally aim to measure/scuttle or judge the action/achievements of decision-makers with a view to improving governance. Our study shows that $62 \%$ of Cameroonian citizens are dissatisfied with the implementation of democracy. This situation is the result of three factors: the perception of the country's economic situation, the current living conditions of citizens and their place of residence (urban and rural). Focusing on these determinants in the development of public policies would undoubtedly in the long run increase citizen satisfaction and ensure the country's development.

Keywords: Satisfaction, democracy, associated factors, governance, Cameroon. 


\section{Introduction Générale}

Selon le rapport de l'Institute for Democracy and Electoral Assistance (IDEA, 2019) sur l'état de la démocratie, $57 \%$ de la population mondiale vit dans une démocratie de même que $67 \%$ des pays du monde sont démocratiques. La démocratie moderne désigne le régime politique mis en place dans les démocraties occidentales postrévolutionnaires.

La démocratie est selon l'Union Interparlementaire, à la fois un idéal et un ensemble d'institutions et de pratiques. En tant qu'idéal, elle exprime deux principes élémentaires : les membres de tout groupe ou association doivent exercer un contrôle sur les règles et les politiques dudit groupe et ces membres doivent avoir entre eux une relation d'égal à égal; en tant qu'ensemble d'institutions et de pratiques, la démocratie inclut: un cadre juridique garantissant le droit des peuples, des institutions dirigeantes représentatives et responsables devant le peuple, un corps citoyen ou une société civile dynamique, des partis politiques qui expriment les opinions et les aspirations des citoyens et des médias qui informent les citoyens dans les affaires publiques.

La satisfaction quant à elle, qui est le paramètre le plus communément utilisé pour mesurer l'état de l'opinion publique concernant le fonctionnement de la démocratie. Elle renvoie à l'idée de performance. Cette perspective est soutenue par les chercheurs qui ont étudié les facteurs déterminants de la satisfaction envers la démocratie. Kornberg \& Clarke, (1994) citent Sartori dans leur texte sur l'appui politique au Canada : « ce qu'est la démocratie ne peut être séparé de ce que la démocratie devrait être ». De même, selon Nadeau (2002), Anderson et Guillory justifient l'usage de cette mesure du fait qu'elle reflète la réalité de la démocratie, notion qui, selon eux, est bien rendue par l'expression « une constitution en état de fonctionnement » adoptée par Lane et Ersonn ou encore par l'expression de Fuchs, GuidoRossi et Svensson, « une réalité constitutionnelle».

\section{Brève revue de littérature et intérêt de l'étude}

Le concept de satisfaction envers la démocratie vise à évaluer la qualité de la démocratie comme le souligne Cutler et al. (2013). Selon ces derniers, ce concept devrait être utilisé dans les démocraties matures plus comme un indicateur d'un jugement subjectif de la qualité de la démocratie. De nombreux auteurs se sont intéressés à cette question à travers divers aspects notamment sur les variables utilisées pour jauger du niveau de satisfaction et les déterminants de la satisfaction envers la démocratie.

Pour ce qui est du type de variables utilisé, on distingue généralement des variables de niveau macro utilisées par des auteurs comme Kornberg et al. (1997) et Anderson \& Singer (2008). L'utilisation des variables de niveau macro a été privilégiée par les auteurs tels qu'Armingeon \& Guthmann (2014).

Quant aux déterminants de la satisfaction envers la démocratie, divers auteurs s'y sont penchés à travers les facteurs comme la performance économique. Il s'agit de Mishler \& Richard (2001), Halla et al. (2013), Armingeon \& Guthmann (2014), Quaranta \& Martini (2016) ; le statut économique abordé par Dahl (1989), Diamond (1992) et Lipset (1994); 
certaines caractéristiques socio-démographiques telles que l'âge et le sexe usités par Crête et al. (1992) et Howe \& Northrup (2000); les conditions économiques et les droits de l'homme abordés par Wike et al. (2019). En outre, certains auteurs ont étudié les variations entre les niveaux de satisfaction. Ces variations sont expliquées selon Anderson \& Guillory (1997) par les caractéristiques individuelles et le contexte institutionnel. D'autres auteurs comme Kaase et al. (1997) ont vu en l'existence d'une crise de légitimité comme cause de l'insatisfaction dans les démocraties occidentales. D'autres auteurs ont utilisé une approche comparative afin d'étudier la satisfaction envers la démocratie. Il s'agit de Nadeau (2002) qui a étudié la satisfaction dans 17 pays en comparaison avec le Canada.

Concernant l'étude de la satisfaction envers la démocratie en Afrique, de nombreux auteurs s'y sont penchés en utilisant par ailleurs les données d'Afrobaromètre. De manière non exhaustive, on peut citer les travaux de (Mattes, 2019) portant sur l'offre et la demande de démocratie en Afrique de manière générale; Alemika (2007) qui analyse la qualité des élections, la satisfaction envers la mise en œuvre de la démocratie et la confiance politique en Afrique; Thioye (2013) a analysé pour le cas du Burkina Faso, la qualité de la gouvernance et de la démocratie en jetant un regard particulier sur la satisfaction envers la démocratie; Chasukwa (2019) quant à lui aborde les différentes faces de la démocratie en s'appesantissant sur la satisfaction et le support envers la démocratie pour le cas du Malawi. Selormey (2019) aborde les aspirations des Africains envers la démocratie et les leçons à en tirer et enfin Møller \& Skaaning (2010) analysent la satisfaction envers la démocratie en Afrique Subsaharienne en mettant un accent particulier sur les déterminants comme la performance du système.

De ce qui précède, on peut dire que la participation des citoyens ou du peuple est donc indispensable et étroitement liée à l'exercice de la démocratie. D'où la nécessité d'une implication effective des citoyens pour l'atteinte des buts ultimes de la démocratie ${ }^{1}$.

La relation entre les citoyens et la mise en œuvre de la démocratie est sujette à des évaluations constantes dans les différentes sociétés (pays). Depuis l'instauration de la démocratie dans les années 1990 dans les pays africains, certains d'entre eux ont favorisé la mise en place d'institutions et du cadre d'épanouissement nécessaire pour vulgariser la démocratie. L'opinion du citoyen occupe donc une place importante dans le système démocratique. Claude (2000) dans son ouvrage intitulé «la faisabilité de la démocratie en Afrique » souligne à juste titre que « lorsque le développement se démocratise de sorte que les gens deviennent en même temps ses agents, ses moyens et sa fin, à ce moment-là le développement sera concrétisé» cité par Wama (2014). En outre, dans son livre «la fin de la pauvreté», Sachs (2005) soutient que la démocratie est une condition préalable au développement économique. Pour lui, un régime despotique, arbitraire et sans foi ni loi peut facilement détruire le développement. Adeolu (2009) quant à lui, observe généralement que

\footnotetext{
${ }^{1}$ La promotion de la dignité et les droits fondamentaux de l'individu, la réalisation de la justice sociale, de favoriser le développement économique et social de la communauté, le renforcement de la cohésion de la société et la création d'un environnement propice à la paix internationale.
} 
«bien qu'aucune société aspirant véritablement au développement ne peut ignorer la démocratie, les expériences démocratiques dans beaucoup de pays du tiers monde laissent un énorme fossé entre les gains attendus de la démocratie et la réalité sur le terrain» (Wama, 2015).

Le Cameroun qui n'échappe pas à cette logique de démocratie a connu différentes étapes avant d'aboutir à sa forme actuelle de démocratie. Ces phases peuvent être divisées en cinq (05) principales à savoir: les périodes coloniales, sous tutelle, l'indépendance et la réunification, de la République Unie du Cameroun à la République du Cameroun et les années 1990 jusqu'à nos jours. Le suivi et la mise en œuvre de cette dernière faisant l'objet d'un suivi particulier au sommet de l'État et d'une satisfaction telle que témoigne les propos du Président de la République Paul BIYA, à la sortie de son vote dans le cadre des législatives en février 2020, «La démocratie fait des pas géants au Cameroun, il y a eu le grand dialogue, il y a les lois sur la décentralisation, le statut spécial, la loi sur le bilinguisme. Et bientôt nous aurons aussi quand les élections municipales auront pris fin, on aura à élire les conseillers régionaux. C'est de grands pas vers une démocratie plus développée. Je n'en suis que satisfait et je remercie les Camerounais d'apporter leur dynamisme, leur concours à la marche de cette grande démocratie $»$.

Ainsi, s'il est vrai que le citoyen a toujours été associé à la question démocratique, a-t-il toujours été satisfait de sa mise en œuvre ? D'où le problème de la relation entre la satisfaction des citoyens et la mise en œuvre de la démocratie de manière générale et le processus de gouvernance en particulier.

Les questions qui structurent notre travail sont donc celles de savoir : quels sont les facteurs qui entrainent la satisfaction des Camerounais envers la démocratie? Quelle appréciation les Camerounais font de la mise en œuvre de la démocratie?

L'objectif général poursuivi dans le présent travail est d'examiner les facteurs associés au niveau de satisfaction des Camerounais envers la démocratie. Il se décline en trois (03) objectifs spécifiques à savoir: appréhender le niveau de satisfaction des citoyens camerounais relativement à la mise en œuvre de la démocratie au Cameroun; analyser l'effet des caractéristiques sociodémographiques (âge, sexe, milieu de résidence, situation économique) sur la satisfaction des citoyens; examiner l'effet de l'intérêt des Camerounais pour les affaires publiques sur le degré de satisfaction relatif à la mise en œuvre de la démocratie.

\section{Démarche méthodologique}

Pour mener à bien notre étude, nous avons utilisé la base de données d'Afrobaromètre Round 7 issu de l'enquête menée au Cameroun en mai 2018. Afrobaromètre est un réseau de chercheurs africains qui mène des projets de recherche d'enquête et de recherche non partisans qui mesurent les attitudes des citoyens sur la démocratie, la gouvernance, l'économie, la société civile et d'autres sujets. Les données issues de ces enquêtes dans environ 34 pays africains sont 
disponibles en accès libre sur la plateforme d'Afrobaromètre ${ }^{2}$. Les échantillons des enquêtes d'Afrobaromètre qui varient entre 1200 et 2400 sont généralement représentatifs de la population du pays avec des taux de réponse de $97 \%$.

\subsection{Les variables utilisées}

- La variable dépendante

La variable dépendante est la satisfaction envers la démocratie. Il s'agit d'une variable ordinale d'échelle allant de 1 à quatre avec les modalités : pas une démocratie, pas du tout satisfait, pas très satisfait, assez satisfait et très satisfait. Cette variable a subi une légère transformation dont le procédé sera décrit plus bas.

- Les variables indépendantes

Quant aux variables indépendantes, on peut les classer en trois groupes : les variables continues telles que l'âge des citoyens; les variables qualitatives binaires à savoir le genre (homme et femme), le milieu de résidence (urbain et rural) et les variables qualitatives ordinales à plus de deux modalités à savoir : l'intérêt pour les affaires politiques (aucun, occasionnel et fréquent), les conditions actuelles des citoyens et la perception de la situation économique du pays avec des échelles de 1 à 5 (très mauvaise, assez mauvaise, ni bonne ni mauvaise, assez bien et très bien).

- Transformations opérées sur les variables utilisées

Aussi l'utilisation des méthodes ci-dessus mentionnées a nécessité la transformation des différentes variables utilisées dans nos analyses. Ainsi l'âge a été regroupé en deux classes à savoir les jeunes (18-55 ans) et les âgés (55 ans à 93 ans). Les variables indépendantes en cinq échelles ont été regroupées en trois modalités (Mauvaise, Bonne et Indifférents). La variable dépendante elle aussi a subi une légère transformation.

\subsection{Les méthodes utilisées}

Différentes méthodes ont été utilisées dans le cadre de ce travail à savoir l'analyse descriptive pour avoir le niveau de satisfaction des individus de même que certaines caractéristiques des citoyens ; une analyse bivariée a été utilisée pour étudier la dépendance ou non entre la variable dépendante et les variables indépendantes à travers le test de Chi 2. Afin de catégoriser les individus, nous avons effectué une Analyse en Correspondance Multiples (ACM). Pour ce qui est de l'analyse multivariée, nous avons opté pour un modèle de régression logistique ordinale compte tenu de la nature de la variable dépendante, mais au préalable nous nous sommes assurés de vérifier l'hypothèse de régression parallèle à travers le test de Brant (Cf. table 15). Pour ce faire, nous avons dichotomisé la variable dépendante (création de $\mathrm{j}+1$ variable) ensuite nous avons procédé au regroupement de certaines variables indépendantes.

\footnotetext{
${ }^{2}$ Les données des différents rounds de même que le processus méthodologique des enquêtes sont disponibles sur le site www.afrobarometer.org
} 
Le seuil de significativité usuel de $5 \%$ a été utilisé pour l'interprétation des résultats. Ces derniers ont été obtenus grâce aux logiciels SPSS 21 et STATA 13.

\section{Les principaux résultats et discussion}

Les résultats de notre étude s'articulent autour de cinq points notamment les caractéristiques de la population, les niveaux de satisfaction et la catégorisation de ces niveaux selon certaines caractéristiques socio-démographiques et enfin les facteurs associés à ces niveaux de satisfaction.

En ce qui concerne les caractéristiques des populations, $20 \%$ des Camerounais discutent régulièrement des affaires politiques, $44 \%$ occasionnellement et $36 \%$ sont indifférents. Par ailleurs l'âge moyen des citoyens camerounais est de 34 ans (avec un âge oscillant entre 18 ans et 93 ans). De même notre échantillon compte 604 hommes et 600 femmes. Une majorité de l'échantillon réside en milieu urbain, soit $53 \%$ contre $47 \%$ en milieu rural. Les Camerounais perçoivent en majorité (51\%) que les conditions économiques de leur pays sont mauvaises contre $36 \%$ qui jugent ces dernières bonnes. Quant à la situation individuelle (actuelle des camerounais) des Camerounais, $42 \%$ estiment que leurs conditions sont bonnes et $40 \%$ estiment que leur situation personnelle était mauvaise (conf. Annexe)

Le deuxième résultat de notre étude qui aborde le niveau de satisfaction des citoyens envers la démocratie indique que seuls $28 \%$ des citoyens camerounais sont satisfaits de la démocratie contre une grande majorité (68 \%) de citoyens insatisfaits (voir tableau en annexe).

Troisièmement, le test d'indépendance du Chi 2 entre le niveau de satisfaction et les certaines caractéristiques socio-démographiques fait ressortir qu'il existe un lien statistiquement significatif au seuil de $5 \%$ entre le niveau de satisfaction et l'âge, le milieu de résidence, l'intérêt pour les affaires politiques, la perception de la situation économique, la situation actuelle (celle de 2018) des citoyens et le genre (voir annexe).

Pour ce qui est de la catégorisation des individus, le graphique de l'ACM ci-dessous nous permet de déterminer la répartition des niveaux de satisfaction à travers les différentes caractéristiques socio-démographiques des citoyens camerounais. Il en ressort que les citoyens satisfaits de la mise en œuvre de la démocratie sont ceux qui avaient une bonne perception de la situation économique du pays et s'intéressent occasionnellement et aucunement aux affaires politiques. Dans cette catégorie on retrouve des citoyens âgés qui résident généralement en milieu rural.

À contrario, tandis que ceux qui considèrent le Cameroun pas comme une démocratie et sont insatisfaits de la mise en œuvre de la démocratie, sont issus du milieu urbain. Ces citoyens s'intéressent fréquemment aux affaires politiques. La perception de ces citoyens de la situation économique du pays est mauvaise de même qu'ils jugent leurs conditions de vie actuelles mauvaises. On y retrouve des femmes et des jeunes. 
Figure 1 : Analyse des Correspondances multiples



Source : auteurs à partir des données afrobaromètre-18

Le tableau de l'ACM nous montre que les deux dimensions représentent $85.3 \%$ de l'inertie totale. Les deux dimensions ( 1 et 2) contribuant respectivement à $64.4 \%$ et $20.9 \%$ de l'inertie totale. La qualité de représentation indique que les modalités satisfaits de la mise en œuvre de la démocratie (0.936), bonne perception de la situation économique du pays $(0.998)$ et bonne situation actuelle (0.968) sont les modalités les mieux représentées. Et les modalités de groupe d'âge sont les plus mal représentées. Quant à la contribution des modalités à la formation des axes, la bonne perception de la situation économique est celle qui contribue le plus à la formation de l'axe 1 (0.349). Pour ce qui est de l'axe 2, la modalité pas de réponse sur la mise en œuvre de la démocratie contribue le plus à la formation de l'axe 2 . 
Tableau 1 : Inerties, coordonnées des dimensions de l'ACM

\begin{tabular}{|c|c|c|c|c|c|c|c|c|c|}
\hline \multirow[b]{2}{*}{ Categories } & \multicolumn{3}{|c|}{ overall } & \multicolumn{3}{|c|}{ dimension_1 } & \multicolumn{3}{|c|}{ dimension_2 } \\
\hline & mass & quality & oinert & coord & sqcorr & contrib & coord & sqcorr & contrib \\
\hline \multicolumn{10}{|l|}{ URBRUR } \\
\hline Urbain & 0.075 & 0.506 & 0.015 & -0.375 & 0.467 & 0.011 & 0.188 & 0.038 & 0.003 \\
\hline Rural & 0.067 & 0.506 & 0.016 & 0.419 & 0.467 & 0.012 & -0.211 & 0.038 & 0.003 \\
\hline \multicolumn{10}{|l|}{ Q101 } \\
\hline Homme & 0.072 & 0.748 & 0.023 & 0.042 & 0.004 & 0.000 & 1.079 & 0.745 & 0.083 \\
\hline Femme & 0.071 & 0.748 & 0.024 & -0.043 & 0.004 & 0.000 & -1.086 & 0.745 & 0.084 \\
\hline \multicolumn{10}{|l|}{ POLDEF } \\
\hline Aucun & 0.051 & 0.875 & 0.042 & 0.141 & 0.015 & 0.001 & -1.845 & 0.859 & 0.172 \\
\hline Occasionnel & 0.063 & 0.413 & 0.011 & 0.113 & 0.045 & 0.001 & 0.564 & 0.367 & 0.020 \\
\hline Frequemment & 0.030 & 0.822 & 0.034 & -0.478 & 0.127 & 0.007 & 1.962 & 0.695 & 0.114 \\
\hline \multicolumn{10}{|l|}{ Satisfaction } \\
\hline pas une de e & 0.003 & 0.641 & 0.005 & -0.912 & 0.326 & 0.003 & 1.571 & 0.315 & 0.008 \\
\hline insatisfa s & 0.089 & 0.764 & 0.027 & -0.585 & 0.717 & 0.030 & 0.260 & 0.046 & 0.006 \\
\hline satisfaits & 0.040 & 0.936 & 0.069 & 1.532 & 0.881 & 0.095 & 0.676 & 0.056 & 0.018 \\
\hline pas de rep e & 0.011 & 0.834 & 0.075 & -0.666 & 0.040 & 0.005 & -5.173 & 0.794 & 0.286 \\
\hline \multicolumn{10}{|l|}{ Perception1 } \\
\hline Mauvaise & 0.073 & 0.973 & 0.160 & -1.799 & 0.958 & 0.237 & 0.390 & 0.015 & 0.011 \\
\hline Bonne & 0.051 & 0.998 & 0.227 & 2.611 & 0.990 & 0.349 & 0.401 & 0.008 & 0.008 \\
\hline Indiffere s & 0.018 & 0.616 & 0.045 & -0.087 & 0.002 & 0.000 & -2.670 & 0.614 & 0.131 \\
\hline \multicolumn{10}{|l|}{ Situation1 } \\
\hline Mauvaise & 0.056 & 0.840 & 0.074 & -1.269 & 0.787 & 0.091 & 0.581 & 0.054 & 0.019 \\
\hline Bonne & 0.060 & 0.968 & 0.095 & 1.541 & 0.967 & 0.143 & -0.067 & 0.001 & 0.000 \\
\hline Indiffere s & 0.026 & 0.469 & 0.037 & -0.795 & 0.292 & 0.017 & -1.086 & 0.177 & 0.031 \\
\hline \multicolumn{10}{|l|}{ AGE2 } \\
\hline Jeunes & 0.127 & 0.022 & 0.002 & -0.012 & 0.005 & 0.000 & 0.037 & 0.017 & 0.000 \\
\hline Agés & 0.016 & 0.022 & 0.018 & 0.096 & 0.005 & 0.000 & -0.301 & 0.017 & 0.001 \\
\hline
\end{tabular}

Source : auteurs à partir des données afrobaromètre-18

Enfin pour ce qui est des facteurs associés, trois facteurs influent sur le niveau de satisfaction des citoyens camerounais envers la démocratie : la perception de la situation économique du pays dont le coefficient positif indique que le niveau de satisfaction augmente au fur à mesure que la perception de la situation économique du pays s'améliore. L'effet marginal signifie que cette augmentation est de 0.141 ; la situation actuelle des citoyens camerounais dont le niveau de satisfaction baisse lorsqu'ils sont indifférents à leur situation (ceux qui ne qualifient pas leurs conditions actuelles). Cette baisse est estimée à 0.345 (effet marginal) et le milieu de résidence dont le coefficient négatif indique que le niveau de 
satisfaction envers la démocratie baisse selon le milieu de résidence (lorsqu'on passe du milieu rural au milieu urbain). Cette baisse est estimée à 0.096 (effet marginal).

Tableau 2 : Résultats des coefficients Ologit

\begin{tabular}{|c|c|c|c|c|c|c|c|c|}
\hline \multicolumn{9}{|c|}{ Iteration 0: log likelihood $=-799.13419$} \\
\hline \multicolumn{9}{|c|}{ Iteration 1: log likelihood $=-741.55812$} \\
\hline \multicolumn{9}{|c|}{ Iteration 2: log likelihood $=-740.42646$} \\
\hline \multicolumn{9}{|c|}{ Iteration 3: log likelihood $=-740.42364$} \\
\hline \multicolumn{9}{|c|}{ Iteration 4: log likelihood $=-740.42364$} \\
\hline \multirow{2}{*}{$\begin{array}{l}\text { Satisfacti } \\
\text { on }\end{array}$} & \multicolumn{2}{|c|}{ Coefficie } & \multirow[t]{2}{*}{ Std.Err } & \multirow[t]{2}{*}{$\mathbf{z}$} & \multirow[b]{2}{*}{$\mathbf{z} \mid$} & $\mathbf{P}>\mathbf{I}$ & \multicolumn{2}{|c|}{$95 \%$ conf.intervall } \\
\hline & nts & & & & & & & \\
\hline \multirow[t]{2}{*}{ Sexe } & & .126031 & 0.13645 & 0.9 & & 0.35 & - & 0.393471 \\
\hline & 9 & & 13 & 2 & 6 & & 0.1414078 & 6 \\
\hline \multirow[t]{2}{*}{ Age } & & .402353 & 0.21370 & 1.8 & & 0.06 & - & .8212042 \\
\hline & 4 & & 33 & 8 & 0 & & 0.0164975 & \\
\hline \multirow[t]{2}{*}{ Politique } & - & & 0.09306 & - & & 0.92 & - & 0.173469 \\
\hline & 0.008 & 89384 & 68 & 0.10 & 3 & & 0.191346 & 1 \\
\hline \multicolumn{9}{|c|}{ Situation Actuelle } \\
\hline \multirow[t]{2}{*}{ Bonne } & & .176697 & 0.16199 & 1.0 & & 0.27 & - & 0.494194 \\
\hline & 6 & & 13 & 9 & 5 & & 0.1407994 & 7 \\
\hline \multirow{2}{*}{$\begin{array}{l}\text { Indiffére } \\
\text { nt }\end{array}$} & - & & 0.20634 & - & & 0.01 & - & - \\
\hline & 0.508 & 87374 & 74 & 2.47 & 4 & & 0.9131709 & 0.1043039 \\
\hline \multicolumn{9}{|l|}{ Perception } \\
\hline \multirow[t]{2}{*}{ Bonne } & & .189517 & 0.15917 & 7.4 & & 0.00 & 0.877538 & 1.501494 \\
\hline & & & 52 & 7 & 0 & & 9 & \\
\hline \multirow{2}{*}{$\begin{array}{l}\text { Indiffére } \\
\text { nt }\end{array}$} & & .854359 & 0.22078 & 3.8 & & 0.00 & 0.421625 & 1.287093 \\
\hline & 2 & & 64 & 7 & 0 & & 8 & \\
\hline \multicolumn{9}{|l|}{ Résidence } \\
\hline \multirow[t]{2}{*}{ urbain } & - & & 0.13429 & - & & 0.00 & - & - \\
\hline & 0.363 & 34594 & 85 & 2.71 & 7 & & 0.6266796 & 0.1002391 \\
\hline \multirow[t]{2}{*}{ /cut1 } & & 3.14834 & 0.37927 & & & & - & - \\
\hline & & & 87 & & & & 3.891712 & 2.404967 \\
\hline \multirow[t]{2}{*}{ /cut2 } & & .728306 & 0.33690 & & & & 1.06799 & 2.388623 \\
\hline & & & 22 & & & & & \\
\hline \multirow{2}{*}{\multicolumn{3}{|c|}{$\begin{array}{c}\text { Nombre } \\
\text { d'observation }=1114\end{array}$}} & Log & \multirow{2}{*}{\multicolumn{2}{|c|}{ likelihood= }} & - & & \\
\hline & & & 740.42364 & & & & & \\
\hline LR chi2 (8) & $=$ & 117.42 & & & & & & \\
\hline Prob > chi2 & $=$ & 0.0000 & & & & & & \\
\hline Pseudo R2 & $=$ & 0.0735 & & & & & & \\
\hline
\end{tabular}

Source : Nos travaux à partir des données d'Afrobaromètre-2018. 


\section{Discussion}

Les résultats sur le niveau de satisfaction des Camerounais envers la mise en œuvre de la démocratie confirment de prime à bord la tendance générale à la baisse de la satisfaction envers la démocratie ces dernières années dans la majorité des pays démocratiques notamment les ÉtatsUnis. Selon le rapport de l'IDEA sur l'état de la démocratie en 2020, les démocraties populaires telles que les États-Unis, le Brésil, le Nigeria et le Mexique ont connu des augmentations des niveaux d'insatisfaction. De manière générale, le niveau de satisfaction des Camerounais est audessus de la moyenne des pays d'Afrique Centrale qui selon Selormey (2019) représente $18 \%$ et en dessous du niveau des pays d'Afrique de l'Ouest ( $46 \%$ selon le même auteur). Ces résultats viennent conforter l'idée généralement avancée par les spécialistes et autres experts sur les questions démocratiques que les pays d'Afrique de l'Ouest ont des processus démocratiques plus évolués que ceux d'Afrique Centrale. Les données d'Afrobaromètre de la même période indiquaient que $51 \%, 58 \%$ et $78 \%$ respectivement de citoyens béninois, burkinabés et ghanéens sont satisfaits de la mise en œuvre de la démocratie. Le faible niveau de satisfaction des Camerounais en ce qui concerne l'offre de la démocratie traduirait de ce fait un réel désir d'amélioration de la gouvernance démocratique. Rappelons cependant qu'en 2018, le Cameroun avait parachevé la mise sur pied de ses institutions démocratiques prévues par la constitution de 1996 notamment le Senat et le Conseil Constitutionnel. Ce qui signifie que l'insatisfaction des Camerounais n'est pas due à l'architecture institutionnelle, mais plutôt à d'autres faits. Aussi le faible niveau de satisfaction des Camerounais envers la démocratie semble conforté par certains indicateurs internationaux sur la démocratie à savoir l'indice global de démocratie ${ }^{3}$. Les scores du Cameroun pour cet indice de 2017 à 2020 étaient respectivement de 3.61, 3.28, 2.85 et 2.77. Ces scores sont en dessous de la moyenne en Afrique subsaharienne (Afrique du Nord exclue) qui était de 4.16 en 2020.

En ce qui concerne la répartition des niveaux de satisfaction envers la démocratie, on peut constater que les Camerounais qui estiment que les conditions économiques du pays sont bonnes sont satisfaits de la mise en œuvre de la démocratie. Ces citoyens s'intéressent occasionnellement aux affaires politiques ce qui traduit probablement le fait que cette classe de citoyens a une bonne connaissance de la vie politique camerounaise et c'est du fait de cet avantage qu'ils émettent un jugement satisfaisant sur la mise en œuvre de la démocratie. Ensuite, le fait que les citoyens âgés et résidant en milieu rural soient satisfaits de la mise en œuvre de la démocratie ne semble pas guère surprenant. En effet les citoyens âgés sont ceux qui sont généralement préoccupés par des problèmes d'autres natures et donc ne semblent plus être intéressés au premier plan par les questions démocratiques. De plus, l'accès de ces personnes à des outils de télécommunications n'est pas toujours aisé à cause des niveaux de désenclavement de ces zones rurales ou de la limitation des infrastructures de télécommunication. Aussi, dans le contexte démocratique en

\footnotetext{
3 Il s'agit d'un indice de l'évaluation du niveau de démocratie des États développé par le magazine the Economist. Cet indice qui varie d'une échelle de 1 à 10 est calculé sur 60 critères qui concernent entre autres : le processus électoral et le pluralisme, les libertés civiles, le fonctionnement du Gouvernement de même que la participation et la culture politique.
} 
Afrique en général et au Cameroun en particulier, le milieu rural est celui où les partis politiques pro-régime ont généralement une forte assise territoriale d'une part ; on y note généralement une quasi-inexistence des autres formations politiques d'autre part. Par ailleurs, la multitude des élites des partis au pouvoir dans les zones rurales est un facteur favorable à la satisfaction envers la mise en œuvre de la démocratie en ce sens que ces derniers à travers les campagnes, des propagandes transmettent une vision édulcorée de la réalité démocratique du pays aux populations. Le milieu urbain étant quant à lui par définition le lieu d'expression d'une multitude d'offres politiques, les citoyens camerounais de ce milieu ont largement accès aux commodités usuelles notamment les communications et les NTIC. Ce qui laisse à supposer qu'ils ont un accès à tous les outils nécessaires pour s'informer de toute l'actualité démocratique camerounaise en particulier et international en général et se faire ainsi leurs propres comparaisons avec les autres pays démocratiques. Et aussi, les actions des partis politiques (qu'ils soient de l'opposition ou du pouvoir) et des autorités gouvernementales sont souvent plus intenses en milieu urbain que rural. Le fait que ces citoyens jugent les conditions du pays mauvaises de même que les leurs peut être dû à ce qu'ils ont une meilleure vision des réalisations économiques du pays de même que diverses réalisations qui se focalisent généralement dans les chefs-lieux de régions. Il parait donc assez logique que ces Camerounais ne soient pas satisfaits de la mise en œuvre de la démocratie et même ne considèrent pas le Cameroun comme une démocratie. Les développements ci-dessus notamment en ce qui concerne l'accessibilité aux NTIC en milieu rural ou urbain peuvent être confortés par les données de l'enquête réalisée par l'Institut National de la Statistique (INS) et l'Agence de Régulation des Télécommunications (ART) portant sur l'accès, les usages et la perception des services de communications électroniques auprès des consommateurs au Cameroun menées en 2014 et concernant l'accès des ménages. Elles nous indiquaient que $53.7 \%$ des ménages en milieu rural disposent d'une radio contre $65.3 \%$ en milieu urbain ; $31.9 \%$ des ménages en milieu rural sont en possession d'un poste de télévision contre $83.9 \%$ en milieu urbain; $25.2 \%$ des ménages du milieu rural disposent d'un téléphone (fixe ou mobile) contre $31.9 \%$ en milieu urbain ; de même $24 \%$ des ménages en milieu rural disposent uniquement d'un téléphone mobile contre $27.6 \%$ en milieu urbain. Pour ce qui est de la possession d'un ordinateur, $3.3 \%$ des ménages en milieu rural en disposent contre $20.7 \%$ en milieu urbain. Enfin pour ce qui est de l'accès à internet à domicile, seul $1.4 \%$ de ménages ruraux en dispose contre $12.3 \%$ en milieu urbain (INS, 2014).

Parlant des facteurs associés notamment la perception de la situation économique du pays et la situation personnelle des Camerounais, ces résultats vont en droite ligne de ceux de Mattes \& Bratton (2000), Wike et al. (2019). Ceci met au gout du jour le débat sur la primauté du bien être économiques sur les considérations démocratiques des citoyens. La seule motivation pour le citoyen serait ainsi son bien-être économique et celui de son pays. En effet dans certains pays communistes, la démocratie qui englobe entre autres les libertés, les droits de l'homme, intérêt capitaliste primerait au détriment des considérations démocratiques. Ainsi les conditions économiques du pays et des citoyens sont les leviers les plus essentiels pour les citoyens camerounais. Ce qui tend à dire que la qualité du régime n'est pas primordiale pour les 
camerounais, du moment où ils ont les garanties d'une bonne vie économique de manière individuelle et si le pays est sur une bonne voie économique. Le résultat le plus intéressant de notre étude réside dans le fait que plus on quitte le milieu urbain, plus les niveaux de satisfaction baissent. En outre, les citoyens attachent ainsi la situation économique de leur pays à leur satisfaction de la mise en œuvre de la démocratie. C'est-à-dire dire que si les conditions économiques étaient meilleures, les citoyens seraient tous satisfaits de la mise en œuvre de la démocratie.

\section{Conclusion générale}

Afin d'appréhender le niveau de satisfaction des citoyens, d'analyser les liens existants entre ce dernier et certaines caractéristiques socio-démographiques et déterminer les facteurs associés au niveau de satisfaction des citoyens camerounais envers la démocratie, nous avons utilisé différentes méthodes notamment univariée, bivariée et explicative cette dernière mettant un ancrage particulier sur une régression logistique ordonnée. Ainsi à travers ces différentes méthodes, des résultats intéressants ont été obtenus qu'il s'agisse de l'insatisfaction des citoyens camerounais envers la démocratie, de la dépendance entre certaines caractéristiques socio-démographiques et le niveau de satisfaction envers la démocratie ou encore des facteurs économiques (perception de la situation économique du pays et individuelle des Camerounais) et du milieu de résidence. Au demeurant, les apports de cet article sont pluriels, de prime abord il s'agit d'une contribution à la littérature scientifique africaine sur les questions de démocratie et de gouvernance; ensuite cette étude se veut pionnière pour ce qui est du Cameroun en ce sens qu'elle allie des méthodes statistiques à la mesure de la gouvernance de manière générale et permet de ce fait de mettre à disposition des dirigeants camerounais, des hommes politiques et des citoyens une grille d'analyse novatrice qui contribuera sans doute pour le Cameroun à améliorer ses idéaux de gouvernance démocratique consignés dans les programmes de références tels que Cameroun émergent à l'horizon 2035 et la Stratégie Nationale de Développement (SND30). De plus, au regard de l'importance de la démocratie dans le processus de développement des divers pays africains, la présente étude est une contribution dans le domaine des politiques publiques en ce sens qu'elle fournit les leviers sur lesquels agir pour améliorer la satisfaction des citoyens camerounais envers l'idéal démocratique. Même si cette contribution scientifique a le mérite d'être actuelle et novatrice en termes de catégorisation des individus, elle présente aussi quelques limites. Premièrement, sur la notion de satisfaction envers la démocratie, qui est une notion assez large, simpliste et récente sur le plan théorique de même qu'elle est très subjective en ce sens qu'il s'agit d'une auto-évaluation des citoyens. Deuxièmement, elle n'a pas tenu compte de certains facteurs notamment ethniques, linguistiques et sociologiques (région d'origine des citoyens) qui affectent le plus souvent les vues des citoyens sur la gouvernance en Afrique en général et au Cameroun en particulier. Aussi, le niveau de satisfaction est mesuré de manière ponctuelle, il serait intéressant de jauger les variations du niveau de satisfaction dans le temps et d'étudier le changement ou non des facteurs associés à la satisfaction envers la démocratie. 


\section{Références :}

Alemika, E. E. (2007). Quality of elections, Satisfaction with democracy and political trust in Africa (Afrobarometer working paper $\mathrm{N}^{\circ} 84$ ).

Anderson, C. J., \& Guillory, C. A. (1997). Political Institutions and Satisfaction with Democracy : A Cross-National Analysis of Consensus and Majoritarian Systems. American Political Science Review, 91(1), 66-81. https://bit.ly/3yv8bdw

Anderson, C. J., \& Singer, M. M. (2008). The Sensitive Left and the Impervious Right. Multilevel Models and the Politics of Inequality, Ideology, and Legitimacy in Europe. Comparative Political Studies, 41(4-5), 564-599.

Armingeon, K., \& Guthmann, K. (2014). Democracy in crisis? The declining support for national democracy in E uropean countries, 2007-2011. European Journal of Political Research, 53(3), 423-442.

Chasukwa, M. (2019). Multiple Faces of Democrats: Satisfaction with Democracy and Support for Democracy in Malawi. Insight on Africa, 11 (1), 18 -37. https://doi.org/10.1177/0975087818814913

Crête, J., Blais, A., Brady, H., \& Johnston, R. (1992). Letting the People Decide: The Dynamics of Canadian Elections. Stanford University Press.

Cutler, F., Nuesser, A., \& Nyblade, B. (2013, septembre 4). Evaluating the quality of democracy with individual level models of satisfaction: or, a complete model of satisfaction with democracy.In ECPR, general conference sciences Po. Bordeaux (pp. 4-7). https://www.scribd.com/document/343817887/textos

Dahl, R. A. (1989). Democracy and its criticis. New Haven: Yale University Press, viii, 397.

Diamond, L. (1992). Economic Development and Democracy Reconsidered. American Behavioral Scientist, 35(4-5), 450-499. https://doi.org/10.1177/000276429203500407

Halla, M., Friedrich, G. S., \& Alexander, F. W. (2013). "Satisfaction with Democracy and Collective Action Problems: The Case of the Environment." Public Choice, 155(1-2), 109-137.

Howe, P., \& Northrup, D. (2000). Strengthening Canadian Democracy: The Views of Canadians. IRPP. https://bit.ly/3m9PAyT

INS. (2014). Quatrième Enquête Camerounaise Auprès des Ménages (p. 145). Institut National de la Statistique (INS). http://nada.stat.cm/index.php

Kaase, M., Newton, K., \& Scarbrough, E. (1997). Beliefs in Government. Politics, 17(2), 135-139. https://doi.org/10.1111/1467-9256.00044

Kornberg, A., \& Clarke, H. D. (1994). Beliefs about Democracy and Satisfaction with Democratic Government : The Canadian Case. Political Research Quarterly, 47(3), 537-563. https://doi.org/10.2307/448843 
Kornberg, R. D., Svejstrup, J. Q., Li, Y., Fellows, J., Gnatt, A., \& Bjorklund, S. (June 1997). Evidence for a mediator cycle at the initiation of transcription. Biochemistry, 94, 6075-6078. https://doi.org/10.1073/pnas.94.12.6075

Lipset, S. M. (1994). Current Revolutions. American Journal of Economics and Sociology, 53(1), 40-40. https://doi.org/10.1111/j.1536-7150.1994.tb02670.x

Mattes, R. (2019). La démocratie en Afrique: La demande, l'offre, et le 'démocrate insatisfait' (Synthèse de Politique $\mathrm{N}^{\mathrm{o}} 54$; p. 32). Afrobaromètre.

Mattes, R., \& Bratton, M. (2000). Democratic and market reforms in Africa: What 'the people' say ( $\mathrm{N}^{\circ}$ 5; Afrobarometer Working paper). Afrobaromètre. https://bit.ly/3mw3Pyf

Mishler, W., \& Richard, R. (2001). Political Support for Incomplete Democracies: Realist vs. Idealist Theories and Measures. International Political Science Review, 22(4), 303-320.

Møller, J., \& Skaaning, S.-E. (2010). Beyond the Radial Delusion : Conceptualizing and Measuring Democracy and Non-democracy. International Political Science Review, 31 (3), 261 -283. https://doi.org/10.1177/0192512110369522

Nadeau, R. (2002). Chapitre 2. La satisfaction envers la démocratie: Le paradoxe canadien. In N. Nevitte (Éd.), Nouvelles valeurs et gouvernance au Canada (p. 41 -72). Presses de l'Université de Montréal. http://books.openedition.org/pum/21410

Quaranta, M., \& Martini, S. (2016). Does the economy really matter for satisfaction with democracy? Longitudinal and cross-country evidence from the European Union. Electoral Studies, 42, 164-174.

Sachs, J. (2005). The end of poverty: How we can make it happen in our lifetime. Penguin.

Selormey, E. E. (2019). Democratic aspirations of Africans : What can we learn from it? (p. 22). Afrobaromètre. https://bit.ly/3dZgaWY

Wama, M. (2014, octobre 10). Afrique : Est-ce que la démocratie garantit le développement? Libre Afrique. https://bit.ly/3E2yMj6

Wike, R., Silver, L., \& Castillo, A. (2019, April 29). Many Across the Globe Are Dissatisfied With How Democracy Is Working : Discontent is tied to concerns about the economy, individual rights and out-of-touch elites (p. 61). Pew Research Center. https://pewrsr.ch/3IYqkF1 


\section{ANNEXES}

\begin{tabular}{|l|l|l|l|}
\hline \multicolumn{3}{|c|}{ Tableau 3 : Répartition des citoyens selon leurs intérêts pour les affaires politiques } \\
\hline Intérêt pour la politique & Effectifs & Percent \\
\hline & Aucun & 427 & 36 \\
\hline & Occasionnel & 527 & 44 \\
\hline & Fréquemment & 250 & 20 \\
\hline & Total & 1204 & 100 \\
\hline
\end{tabular}

Source : auteurs à partir des données afrobaromètre-18

Tableau 4 : statistiques descriptives de l'âge

$\begin{array}{ll}\text { Valide } & 1203 \\ \text { Manquant } & 1\end{array}$

Moyenne 34

Médiane 30

Écart-Type 14

Variance 207

Minimum 18

Maximum 93

Source : auteurs à partir des données afrobaromètre-18

\begin{tabular}{|c|c|c|}
\hline Genre & Effectif & Pourcentage \\
\hline homme & 604 & 50,2 \\
\hline femme & 600 & 49,8 \\
\hline Total & 1204 & 100 \\
\hline
\end{tabular}

Source : auteurs à partir des données afrobaromètre-18

\begin{tabular}{lll}
\hline \multicolumn{3}{l}{ Tableau 6 $:$ répartition des citoyens selon le milieu de résidence } \\
\hline Résidence & Effectif & Pourcentage \\
\hline Urbain & 636 & 53 \\
Rural & 568 & 47 \\
\hline Total & 1204 & 100 \\
\hline \multicolumn{2}{c}{ Source $:$ auteurs à partir des données afrobaromètre-18 }
\end{tabular}

\begin{tabular}{llc}
\hline Tableau 7 : répartition des citoyens selon la perception de l'économie \\
\hline Perception & Effectif & Pourcentage \\
\hline mauvaise & 618 & 51 \\
\hline bonne & 431 & 36 \\
\hline indifférents & 155 & 13 \\
\hline Total & 1204 & 100 \\
\hline
\end{tabular}

Source : auteurs à partir des données afrobaromètre-2018 


\begin{tabular}{lll}
\hline \multicolumn{2}{c}{ Tableau 8 : répartition des citoyens selon le niveau satisfaction } \\
\hline Niveau de satisfaction & Effectif & Pourcentage \\
\hline Pas une démocratie & 26 & 2 \\
Insatisfaits & 748 & 62 \\
Satisfaits & 340 & 28 \\
pas de réponse & 90 & 8 \\
\hline Total & 1204 & 100 \\
\hline
\end{tabular}

Source : auteurs à partir des données afrobaromètre-18

Tableau 9 : Répartition des citoyens selon le niveau de satisfaction et le milieu de résidence

\begin{tabular}{llrrc}
\hline & & \multicolumn{2}{c}{ Résidence } & \multirow{2}{*}{ Total } \\
& & Urbain & Rural & \\
\hline \multirow{3}{*}{ Satisfaction } & Pas une démocratie & 2 & 2 & 2 \\
& Insatisfaits & 70 & 55 & 62 \\
& Satisfaits & 23 & 34 & 28 \\
& pas de réponse & 5 & 9 & 8 \\
\hline Total & & 100 & 100 & 100 \\
\hline
\end{tabular}

\begin{tabular}{lccc}
\multicolumn{4}{c}{ Chi-Square Tests } \\
\hline & Value & df & Asymp. Sig. (2-sided) \\
\hline Pearson Chi-2 & 26.33 & 3 & .000 \\
Rapport de Vraisemblance & 26.38 & 3 & .000 \\
Association linéaire par linéaire & 19.12 & 1 & .000 \\
Observations valides & 1204 & & \\
\hline
\end{tabular}

Source : auteurs à partir des données afrobaromètre-18

Tableau 10 : répartition des citoyens selon le niveau de satisfaction et l'intérêt pour les affaires politiques

\begin{tabular}{llcccc}
\hline & & \multicolumn{3}{c}{ Intérêt politique } & \multirow{2}{*}{ Total } \\
& & Aucun & Occasionnel & Fréquemment & \\
\hline \multirow{4}{*}{ Satisfaction } & Pas une démocratie & 2 & 2 & 3 & 2 \\
& Insatisfaits & 56 & 66 & 64 & 62 \\
& Satisfaits & 27 & 29 & 29 & 28 \\
& pas de réponse & 15 & 3 & 4 & 8 \\
\hline Total & & 100 & 100 & 100 & 100 \\
\hline
\end{tabular}

Chi-Square Tests

\begin{tabular}{lrrc}
\hline & Value & df & Asymp. Sig. (2-sided) \\
\hline Pearson Chi-2 & 54.80 & 6 & .000 \\
Rapport de Vraisemblance & 52.18 & 6 & .000 \\
Association linéaire par linéaire & 21.69 & 1 & .000 \\
Observations valides & 1204 & & \\
\hline
\end{tabular}

Source : auteurs à partir des données afrobaromètre-18 


\begin{tabular}{llccc}
\hline Tableau 11 : répartition des citoyens selon le niveau de satisfaction et le genre \\
\hline \multirow{4}{*}{ Satisfaction } & \multicolumn{2}{c}{ genre } & Total \\
& Pas une démocratie & 2 & 2 & 2 \\
& Insatisfaits & 62 & 62 & 62 \\
& Satisfaits & 31 & 26 & 28 \\
& pas de réponse & 5 & 10 & 8 \\
\hline Total & & 100 & 100 & 100 \\
\hline
\end{tabular}

\begin{tabular}{lccc}
\multicolumn{4}{c}{ Chi-Square Tests } \\
\hline & Value & df & Asymp. Sig. (2-sided) \\
\hline Pearson Chi-2 & 14.89 & 3 & .002 \\
Rapport de Vraisemblance & 13.60 & 3 & .004 \\
Association linéaire par linéaire & 14.09 & 1 & .000 \\
Observation valides & 1204 & & \\
\hline
\end{tabular}

Source : auteurs à partir des données afrobaromètre-18

Tableau 12 : répartition des citoyens selon le niveau de satisfaction et la perception de l'économie du pays

\begin{tabular}{llcccc}
\hline & & \multicolumn{3}{c}{ perception économie } & \multirow{2}{*}{ Total } \\
& Pas une démocratie & 3 & 1 & 1 & 2 \\
\multirow{3}{*}{ Satisfaction } & Bonne & Indifférents & \\
& Insatisfaits & 73 & 49 & 55 & 62 \\
& Satisfaits & 17 & 45 & 27 & 28 \\
& pas de réponse & 7 & 5 & 17 & 8 \\
\hline
\end{tabular}

Source : auteurs à partir des données afrobaromètre-18

Tableau 13 : répartition des citoyens selon le niveau de satisfaction et la situation actuelle

\begin{tabular}{|c|c|c|c|c|c|c|}
\hline & & \multicolumn{4}{|c|}{ Situation actuelle } & \multirow{2}{*}{ Tota } \\
\hline & & \multicolumn{2}{|c|}{ Mauvaise } & Bonne & Indifférents & \\
\hline \multirow{5}{*}{ Satisfaction } & Pas une démocratie & & 3 & 2 & 2 & 2 \\
\hline & Insatisfaits & & 65 & 55 & 72 & 62 \\
\hline & Satisfaits & & 24 & 38 & 17 & 28 \\
\hline & pas de réponse & & 9 & 5 & 9 & 8 \\
\hline & \multicolumn{3}{|c|}{ Total } & 100 & 100 & 100 \\
\hline \multicolumn{6}{|c|}{ Chi-Square Tests } & \\
\hline & & Value & df & \multicolumn{2}{|c|}{ Asymp. Sig. (2-sided) } & \\
\hline \multicolumn{2}{|c|}{ Pearson Chi-2 } & 123.09 & 6 & \multicolumn{2}{|c|}{.000} & \\
\hline \multicolumn{2}{|c|}{ Rapport de Vraisemblance } & 116.99 & 6 & \multicolumn{2}{|c|}{.000} & \\
\hline \multicolumn{2}{|c|}{ Association linéaire par linéaire } & 48.44 & 1 & \multicolumn{2}{|c|}{.000} & \\
\hline \multicolumn{2}{|c|}{ Observations valides } & 1204 & & & & \\
\hline
\end{tabular}

Source : auteurs à partir des données afrobaromètre-18 
Tableau 14 : répartition des citoyens selon le niveau de satisfaction et le genre

\begin{tabular}{llccc}
\hline & & \multicolumn{2}{c}{ Age } & \multirow{2}{*}{ Jeunes } \\
& Pas une démocratie & 2 & 1 & Aotal \\
\hline \multirow{3}{*}{ Satisfaction } & 63 & 52 & 62 \\
& Insatisfaits & 28 & 33 & 28 \\
& Satisfaits & 7 & 14 & 8 \\
\hline & pas de réponse & & 100 & 100 \\
\hline
\end{tabular}

Chi-Square Tests

\begin{tabular}{lrrc}
\hline & Value & df & Asymp. Sig. (2-sided) \\
\hline Pearson Chi-2 & 14,89 & 3 & .002 \\
Rapport de Vraisemblance & 13,60 & 3 & .004 \\
Association linéaire par linéaire & 14,09 & 1 & .000 \\
Observation valides & 1204 & & \\
\hline
\end{tabular}

Source : auteurs à partir des données afrobaromètre-18

Tableau 15 : Test de Brant 2

Brant test of parallel regression assumption

\begin{tabular}{l|l|l|r}
\hline & chi2 & p>chi2 & df \\
\hline All & 8.12 & 0.229 & 6 \\
\hline Politique & 0.43 & 0.512 & 1 \\
\hline Perception & 0.05 & 0.826 & 1 \\
\hline Situation & 1.72 & 0.189 & 1 \\
\hline Sexe & 0.01 & 0.940 & 1 \\
\hline Age & 0.48 & 0.489 & 1 \\
\hline Résidence & 5.03 & 0.025 & 1 \\
\hline
\end{tabular}

Le test statistique significatif fournit l'évidence que

l'hypothèse de régression parallèle a été violée.

Source : Nos travaux à partir des données d'Afrobaromètre-2018.

Tableau 16 : Effets marginaux

Effets Marginaux après ologit

$\mathrm{Y}=\operatorname{pr}($ satis $=3)($ predict, $\mathrm{p}$ outcome $(3))$

\begin{tabular}{l|l}
\hline 0,29335781 & $\mathrm{dy} / \mathrm{dx}$ \\
\hline Variables & -0.345382 \\
\hline Situation actuelle & 0.1413039 \\
\hline Perception & -0.0963174 \\
\hline Milieu de résidence
\end{tabular}

Source : Nos travaux à partir des données d'Afrobaromètre-2018 